\title{
Adsorption of Olive Leaf (Olea europaea L.) Antioxidants on Silk Fibroin
}

\author{
Deniz Bayçın, ${ }^{\dagger}$ Evren Altiok, Semra Ülkü, And Oguz BayrakTar**
}

Biochemical Engineering Research Laboratory (BERL), Department of Chemical Engineering, Izmir Institute of Technology, Gülbahçe Köyü, 35430 Urla-Izmir, Turkey

\begin{abstract}
The adsorption isotherms of oleuropein and rutin were evaluated at different temperatures, $\mathrm{pH}$ values, and solid/liquid ratios. The experimental data of adsorption isotherms were well fitted to a Langmuir model. The maximum adsorption capacities were determined as $108 \mathrm{mg}$ of oleuropein $/ \mathrm{g}$ of silk fibroin and $21 \mathrm{mg}$ of rutin/g of silk fibroin. After adsorption of oleuropein and rutin, the antioxidant capacity of silk fibroin increased from 1.93 to $3.61 \mathrm{mmol}$ of TEAC/g. Silk fibroin also gained antimicrobial activity against Staphylococcus aureus and Klebsiella pneumoniae after adsorption of olive leaf antioxidants. In a desorption process, $81 \%$ of rutin and $85 \%$ of oleuropein were removed from the adsorbent surface in $70 \%$ aqueous ethanol solution. Consequently, silk fibroin was found to be a promising biomaterial for the production of functional food or dietary supplements and for the purification of oleuropein and rutin from olive leaf extracts.
\end{abstract}

KEYWORDS: Olea europaea; silk fibroin; olive leaves; antioxidant; antimicrobial; oleuropein; rutin

\section{INTRODUCTION}

In recent years, there is a growing interest in obtaining biologically active compounds from natural sources. The potential dangers of some synthetic antioxidants such as butylated hydroxytoluene (BHT) and butylated hydroxyanisole (BHA) have been documented, and this has stimulated the substitution of synthetic antioxidants by natural ones $(1,2)$. Phytochemicals, especially polyphenols in plants, are the major bioactive compounds because of their antioxidative, antimicrobial, antiproliferative, antiviral, and antiinflammatory properties $(3-5)$.

The olive tree is one of the potential natural antioxidant sources because of its phenolic content. The olive leaf has been known as a symbol of the Mediterranean region and peace since ancient times $(2,6,7)$. Historically, the olive leaf has been used as a folk remedy for several diseases such as fevers and malaria (7). The most abundant and bioactive components of olive leaf are oleuropein and rutin. The health-promoting properties of oleuropein and rutin are widely investigated in the literature $(6,8-11)$.

Due to the special health-promoting and disease-preventing effects of polyphenols, efficient methods for extracting polyphenols from different plants have been developed (12). Many plant species have been investigated for their novel antioxidants, and some natural antioxidants such as rosemary and sage are already exploited commercially either as antioxidant additives or as nutritional supplements (13).

* Author to whom correspondence should be addressed (telephone +90 232 750-6657; fax +90 232 750-6645; e-mail oguzbayraktar@iyte.edu.tr).

† Present address: Department of Chemical and Biomolecular Engineering, 221 Maryland Hall, Johns Hopkins University, 3400 N. Charles St., Baltimore, MD 21218.
Although the organic solvents provide the extraction of the metabolites from plants, further purification can be essential in order to obtain concentrated specific components selectively because many other compounds such as sugars, protein, or metals may exist in the plant extracts $(1,14)$. For the selective recovery of target plant metabolites from the crude solvent extracts, adsorption is preferred by many of the researchers, because it is a low-cost separation technique (14). For the further purification of the polyphenols, adsorption and desorption processes can take place, respectively. The adsorption process is also used for the removal of undesired compounds from the plant extracts. Moreover, because of the worldwide tendency to replace synthetic colorants with natural pigments, anthocyanins, which are responsible for red, blue, and purple colors, have received growing attention as European food colorants and, agaih, adsorption is the preferred technique to recover these flavonoids, especially from citrus processing (15).

Ribeiro et al. (16) studied the removal of limonin and naringin from citrus juices becacuse these flavonone glycosides cause the bitterness of orange juices. Activated diatomaceous earths, granulated activated carbon, and synthetic neutral resins (Amberlite XAD-4, XAD-7, and XAD-16) were used as adsorbents. They observed a favorable adsorption of limonin and naringin onto neutral resins (XAD-4 and XAD-7) compared to the other components such as carotenoids and sugars in citrus juices because hydrophobic interactions played a great role in the adsorption of limonin and naringin (apolar solutes) from aqueous solution onto neutral adsorbents (16).

Many biopolymers have been used as adsorbents for the recovery of polyphenols. The interaction between the biopolymers and antioxidative polyphenols has been widely investigated (17). Cellulose and collagen are nearly the most common 
biopolymer used as an adsorbent for the purification of plant extracts. For instance, some researchers achieved $93.8 \%$ recovery of trans-resveratrol, an important natural polyphenol found in grapes, on cellulose cotton by increasing the salt concentration and decreasing the temperature of the solution (18). There is an increasing trend to purify many other polyphenols from plants, which have antioxidative properties. Because of their promising health effects, oleuropein and rutin are the two essential components of the olive leaf. They can be removed from crude olive leaf extract by adsorption techniques. If an edible and medicinal adsorbent such as silk fibroin can be used instead of these commercial resins in the adsorption of oleuropein and rutin, then there will be no need for a desorption step, and in this way silk fibroin will gain antioxidative, antimicrobial, and anti-inflammatory properties for further use in industry as functional food or dietary supplement.

Silk fibroin is a protein polymer (19) that consists of highly repetitive regions. Silk fibroin is an edible protein, which has functional amino acids in its structure, and it is preferred in many biotechnological applications such as drug delivery and tissue engineering (20-22). It has also been used as an adsorbent since the 1940 s because of its hydrophobic and bonding characteristics $(23-25)$. Silk fibroin can be considered as a potent adsorbent for the isolation of antioxidants from the crude extracts, which will increase its health benefits. However, silk fibroin has not been used as an adsorbent for the removal of any polyphenolic structures.

This study focused on the production of protein-based functional food with antioxidative and antimicrobial properties. To our knowledge, in the literature there were no studies about the adsorption of olive leaf polyphenols on silk fibroin or on any adsorbents. Therefore, extraction and adsorption of the two most abundant polyphenols in olive leaves, oleuropein and rutin, on silk fibroin were studied. This study also aimed to increase the purity of oleuropein and rutin. For this reason, desorption studies were performed after adsorption to understand whether the silk fibroin can be used as a purification material for these polyphenols.

\section{MATERIALS AND METHODS}

Plant Material, Reagents, and Standards. Fresh green olive leaves were collected from the olive trees grown on the campus of Izmir Institute of Technology (Izmir, Turkey). Field collections were made in December 2004. Oleuropein and coumarin (internal standard) were obtained from Extrasynthese (Genay, France), whereas rutin $(98.5 \%)$ was obtained from Merck Co. (Darmstadt, Germany). All solvents were of high-performance liquid chromatography (HPLC) grade. Ethanol (for extraction) from Riedel-de Haën (Seelze, Germany), acetonitrile (mobile phase for HPLC) from Sigma-Aldrich Chemie (Steinheim, Germany), and acetic acid (mobile phase for HPLC) from Merck Co. were used in this study. Citric acid monohydrate (for phosphate buffer) was supplied from Sigma-Aldrich Chemie (Steinheim, Germany), and disodium hydrogen phosphate $(99 \%)$ (for phosphate buffer) was supplied from Merck Co. 2,2'-Azinobis(3-ethylbenzothiazoline-6sulfonic acid) (ABTS) from Sigma (Steinheim, Germany), Trolox (6hydroxy-2,5,7,8,-tetramethylchroman-2-carboxylic acid), and potassium persulfate $\left(\mathrm{K}_{2} \mathrm{O}_{8} \mathrm{H}_{8}\right)$ from Fluka (Steinheim, Germany) were used for antioxidant analysis. Hydrophobic silk fibroin from Silk Biochemical Co., Ltd. (Hangzhou, China), was used as adsorbent to determine the adsorption behavior of olive leaf antioxidants on it.

Preparation of Crude Extracts from Olive Leaves. Collected olive leaves were washed with deionized water and then dried at $37^{\circ} \mathrm{C}$ for 3 consecutive days. The dried leaves were powdered and extracted in $70 \%$ ethanol aqueous solution for $2 \mathrm{~h}$ at $25{ }^{\circ} \mathrm{C}$. The solvent of the extracted medium was removed by using rotary evaporator at $38{ }^{\circ} \mathrm{C}$, $120 \mathrm{rpm}$ rotation under vacuum. Then, the solvent-free olive leaf extract was dried using a freeze-dryer system at $-52{ }^{\circ} \mathrm{C}$ and $0.2 \mathrm{mbar}$, and it was stored in light-protected glasses until further use in adsorption studies.

Adsorption and Desorption of Olive Leaf Antioxidants. Crude olive leaf extract was dissolved in proper solvent and mixed with silk fibroin in a batch system for the adsorption process. The adsorption process was performed in a thermoshaker at $250 \mathrm{rpm}$ for $30 \mathrm{~h}$. Several parameters were measured in the adsorption procedure. The solvent effect on adsorption was investigated by using a $70 \%$ ethanol-water solution and water. Then the adsorption kinetics of this system was followed during $30 \mathrm{~h}$ at $25^{\circ} \mathrm{C}$ when the solid/liquid ratio was 1:20. The adsorption behavior of the system at different concentrations was investigated to plot the adsorption isotherms. The experiments were run at three different temperatures, 25,30 , and $35^{\circ} \mathrm{C}$, to show the effect of temperature on the adsorption isotherms. At constant temperature, the $\mathrm{pH}$ of the solution was adjusted to $2.4,4.0$, and 7.6 by using citric acid monohydrate and phosphate buffer. Also, water at $\mathrm{pH}$ 6.0 was used to see its effect on adsorption isotherms. Then at constant $\mathrm{pH}$ and temperature, the solid/liquid ratio was changed from 0.025 to 0.1 to demonstrate its effect on the adsorption.

After adsorption, the samples were centrifuged at $8000 \mathrm{rpm}$ for 15 min. Then the liquid and solid were separated. After centrifugation, the supernatant of the samples before and after adsorption was analyzed by using HPLC. The results of HPLC provided data to plot the adsorption isotherms of oleuropein and rutin at different initial concentrations and at different conditions. After adsorption, the dried remaining silk fibroin powder containing olive leaf constituents was mixed with $70 \%$ ethanol to perform the desorption. The desorption process was performed in the thermoshaker at $250 \mathrm{rpm}$ and $25^{\circ} \mathrm{C}$ during 4 days. Then the mixture was centrifuged to separate the liquid phase from the solid phase. The supernatant was analyzed by using HPLC to determine the desorption and purification efficiency of this system. Furthermore, the antioxidant capacity of this sample was measured to see whether the olive leaf flavonoids still had antioxidant activity after adsorption.

FTIR analyses of dried silk fibroin before and after adsorption process were carried out in the region of $500-7000 \mathrm{~cm}^{-1}$ using an FTIR spectrophotometer (Digilab FTS $3000 \mathrm{Mx}$ ).

HPLC Analysis. The HPLC analysis given in the literature (7) was used for the quantification of oleuropein and rutin. The HPLC equipment used was a Hewlett-Packard series HP 1100 equipped with a diode array detector. The stationary phase was a C18 LiChrospher 100 analytical column $(250 \times 4 \mathrm{~mm}$ i.d. $)$ with a particle size of $5 \mu \mathrm{m}$ thermostated at $30^{\circ} \mathrm{C}$. The flow rate was $1 \mathrm{~mL} / \mathrm{min}$, and the absorbance changes were monitored at $280 \mathrm{~nm}$. The mobile phases for chromatographic analysis were (A) acetic acid/water (2.5:97.5) and (B) acetonitrile. A linear gradient was run from $95 \% \mathrm{~A}$ and $5 \% \mathrm{~B}$ to $75 \% \mathrm{~A}$ and $25 \% \mathrm{~B}$ during $20 \mathrm{~min}$; it changed to $50 \% \mathrm{~A}$ and $\mathrm{B}$ in $20 \mathrm{~min}(40$ min, total time); in $10 \mathrm{~min}$ it changed to $20 \% \mathrm{~A}$ and $80 \% \mathrm{~B}$ (50 min, total time), after reequilibration in $10 \mathrm{~min}$ ( $60 \mathrm{~min}$, total time) to initial composition. Oleuropein and rutin in olive leaf extracts were identified by comparison of their retention times with the corresponding standards. Coumarin was used as internal standard for the quantification of oleuropein and rutin. Because the retention time of coumarin is between the retention times of oleuropein and rutin and because it does not react with the polyphenols in olive leaf extract, coumarin was preferred as an internal standard in this study.

Total Antioxidant Activity Analysis. The antioxidant analysis method was based on the ability of olive leaf antioxidants to scavenge the $\mathrm{ABTS}^{\bullet+}$ (ABTS radical cation) compared with a standard antioxidant (Trolox) in a dose-response curve (26). A $7 \mathrm{mM}$ aqueous ABTS solution was mixed with $2.45 \mathrm{mM}$ potassium persulfate solution to form $\mathrm{ABTS}^{\bullet+}$. The mixture was kept in the dark at ambient temperature for $12-16 \mathrm{~h}$ to complete the reaction. The $\mathrm{ABTS}^{\bullet+}$ solution was diluted with ethanol to an absorbance of $0.7( \pm 0.02)$ at $734 \mathrm{~nm}$ and equilibrated at $30{ }^{\circ} \mathrm{C}$. The samples of silk fibroin before and after adsorption, which were prepared by dissolving them in Ajisawa's reagent $\left(\mathrm{CaCl}_{2} / \mathrm{ethanol} /\right.$ water, 111:92:144 in weight) (27), and olive leaf extract solutions were analyzed for their antioxidant capacities. Twenty microliters of samples was added to $2 \mathrm{~mL}$ of diluted $\mathrm{ABTS}^{+}$solution, and the absorbance was taken every minute during 6 min with a UV-visible spectropho- 


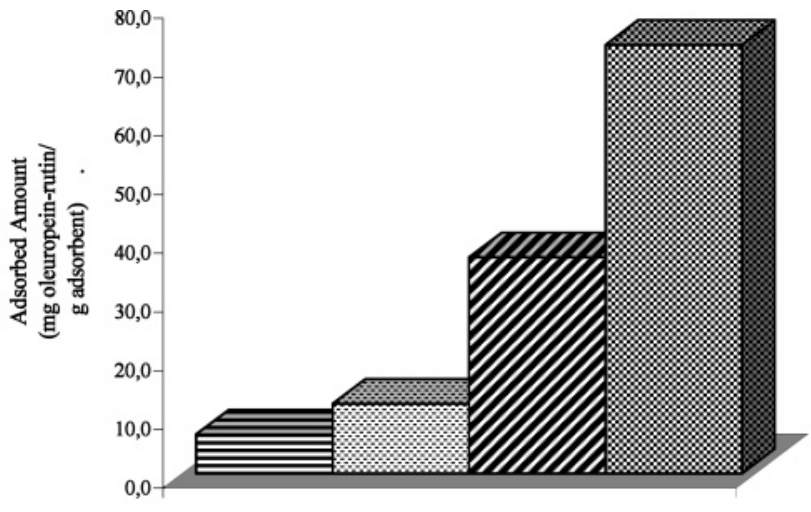

\begin{tabular}{|ll|}
\hline 日rutin (70 \% ethanol) & Q rutin (water) \\
Doleuropein (70\% ethanol) & 叉 oleuropein (water) \\
\hline
\end{tabular}

Figure 1. Adsorbed amount of rutin and oleuropein on silk fibroin in $70 \%$ aqueous ethanol solution and water.

tometer (Perkin-Elmer-Lambda 25). All samples were analyzed at least three times at different concentrations. Five different concentrations of each sample were analyzed to obtain 20-80\% inhibition in absorbance of the $\mathrm{ABTS}^{-+}$solution. The graph between the concentration of sample and percentage inhibition in $\mathrm{ABTS}^{\bullet+}$ solution was obtained, and the slope of this plot was compared with the standard Trolox curve's slope to determine TEAC value.

Antimicrobial Tests. Antimicrobial tests were performed to determine the effect of olive leaf extract against the growth of certain bacteria. Solid powder samples were pressed in a hydraulic press machine to form pellets with a diameter of $14 \mathrm{~mm}$. The antibacterial action of these four samples against Escherichia coli, Staphylococcus aureus, Klebsiella pneumoniae, and Pseudomonas aeruginosa was determined by the disk diffusion method. Bacterial cultures were grown on Mueller-Hinton agar medium at $37{ }^{\circ} \mathrm{C}$ overnight. One of the colonies was picked up and dissolved in $1 \mathrm{~mL}$ of phosphate buffer solution (PBS); the turbidity was adjusted to McFarland no. $1\left(10^{9}\right.$ $\mathrm{CFU}$ ). A sterile cotton swab was placed in the bacterial suspension and then streaked in three directions over the surface of the MuellerHinton agar to obtain uniform growth. Four different disks or pellets were placed into the plates of each bacterium. The plates were incubated at $37^{\circ} \mathrm{C}$ for 1 day. Besides this, three commercial antibiotics, penicillin, vancomycin, and gentamicin pellets of $6 \mathrm{~mm}$ diameter, were placed in each plate of the bacterium for positive control. Then the width of the inhibition zone of each sample and commercial antibiotics in the plates was measured.

\section{RESULTS AND DISCUSSION}

Effect of Solvent Type on Adsorption. The olive leaf extract was analyzed by using HPLC before and after adsorption to determine the concentration of oleuropein and rutin in the liquid phase and the adsorbed amounts on silk fibroin. Adsorption on silk fibroin powder was performed in $70 \%$ aqueous ethanol solution and deionized water. After adsorption, there were visible changes in the color of the silk fibroin. The color of the silk fibroin turned to light green from white when the adsorption was carried out in $70 \%$ aqueous ethanol solution, and it turned to dark yellow when the adsorption was performed in water. Figure 1 represents the adsorbed quantities of oleuropein and rutin per gram of adsorbent in different solvents.

The highest quantities of oleuropein and rutin were adsorbed in water, so the adsorption was more efficiently performed with water compared with $70 \%$ aqueous ethanol solution. For this reason, all of the other adsorption experiments were performed in water. The higher adsorption efficiency in water showed that probably hydrophobic interactions take place between the olive leaf antioxidants and silk fibroin. The interactions between the biopolymers and polyphenols are mainly hydrophobic interactions and hydrogen bonding. In hydrophilic solvents, hydrophobic interactions are favored, whereas in hydrophobic solvents, hydrogen bonding is favored (17).

To understand whether the silk fibroin selectively adsorbed the rutin and oleuropein, the two HPLC chromatograms before adsorption and after adsorption were overlapped in a graph. Figure 2 proves this selective adsorption of oleuropein and rutin on silk fibroin. The selective accumulation of rutin and oleuropein on the hydrophobic silk fibroin revealed the presence of interaction between silk fibroin and polyphenols during the adsorption process. The other polyphenols in olive leaf extract such as verbascoside, apigenin-7-glucoside, and diosmetin-7glucoside, which have retention times closer to the retention times of rutin and oleuropein, were also adsorbed on silk fibroin. However, the amount of these antioxidants in olive leaf extract and the adsorbed amount of them on silk fibroin are less compared to the adsorbed amounts of oleuropein and rutin. All

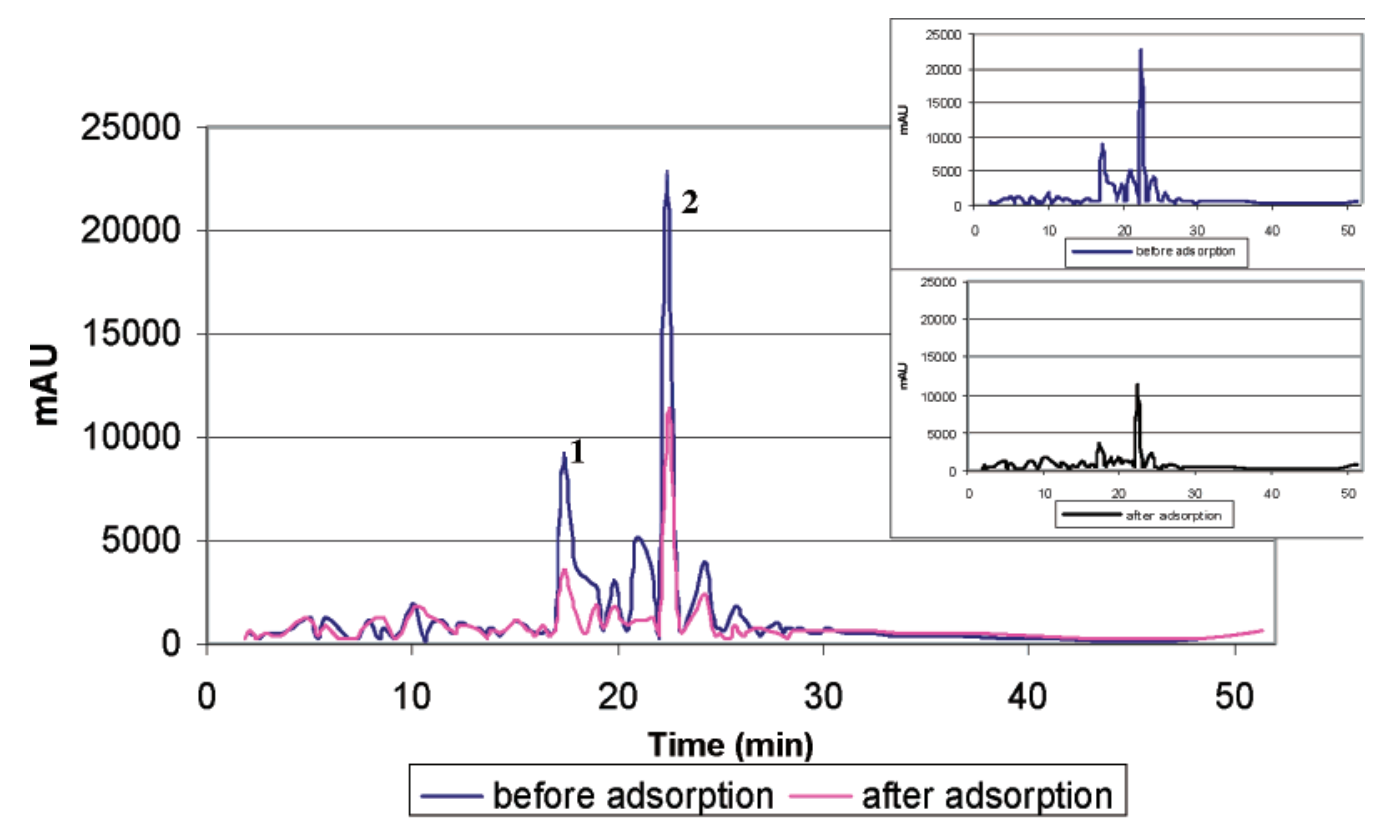

Figure 2. HPLC chromatograms of olive leaf extract before and after adsorption: (1) rutin peak; (2) oleuropein peak. 


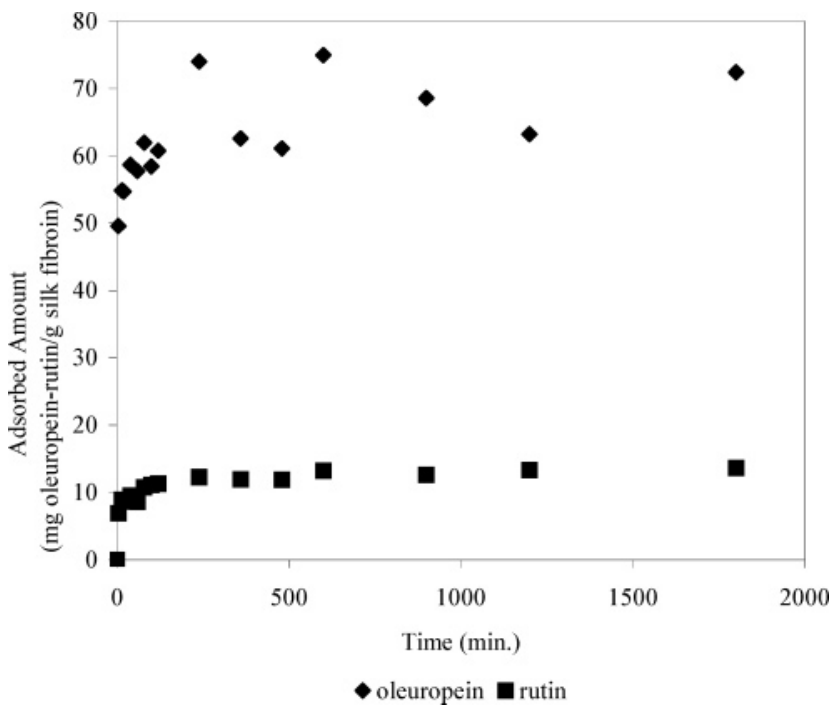

Figure 3. Adsorption behavior of oleuropein and rutin on silk fibroin during $30 \mathrm{~h}$.

of these polyphenols possess high antioxidative activity, which increases the antioxidant capacity of this protein-based functional food.

Adsorption Equilibrium. The adsorption behavior of oleuropein and rutin in water was followed during $30 \mathrm{~h}$ by running each experiment as three replicates. Figure 3 shows the adsorbed amount of oleuropein and rutin depending on time. The adsorption significantly occurred in the first $5 \mathrm{~min}$, and then the adsorbed quantity slightly increased in $40 \mathrm{~min}$. After 40 $\mathrm{min}$, the adsorption reached equilibrium. Consequently, $40 \mathrm{~min}$ was sufficient for the removal of olive leaf antioxidants, and after $40 \mathrm{~min}$ of contact time, the steady-state assumption was valid for this system. The studies in the literature established that approximately 2 or $3 \mathrm{~h}$ of adsorption time was generally adequate for the recovery of polyphenols $(15,16,28,29)$. The equilibrium time has great importance in finding the maximum adsorption capacity of the system and in performing adsorption isotherms.

Adsorption Isotherms of Oleuropein and Rutin. Adsorption isotherms are the best systems that describe the interactions and the maximum capacity of the adsorbents. For this reason, adsorption isotherms of oleuropein and rutin at different conditions were established, successively.

Oleuropein is the most abundant compound in the olive leaf. Because of this and its relatively nonpolar character, a high amount of oleuropein was adsorbed on silk fibroin. To find the maximum capacity of silk fibroin for the adsorption of oleuropein, the adsorption was accomplished at different initial concentrations. Figure 4 shows the effect of initial concentration on the adsorption of oleuropein, and the adsorption isotherm of oleuropein at $25^{\circ} \mathrm{C}$ is given in this figure.

An increase in the concentration of oleuropein in the solution was achieved by increasing the amount of olive leaf extract dissolved. The initial concentration of oleuropein was changed from 0.79 to $9.03 \mathrm{mg} / \mathrm{mL}$. A significant increase in the adsorption of oleuropein was observed until the initial concentration of oleuropein was set to $6.72 \mathrm{mg} / \mathrm{mL}$, and concentrations over $6.72 \mathrm{mg} / \mathrm{mL}$ did not affect the adsorption of oleuropein.

Langmuir and Freundlich models were applied to equilibrium data of the adsorption isotherm. The Langmuir model demonstrated considerable superiority to the Freundlich model because there is a limit in the adsorption capacity of silk fibroin. The

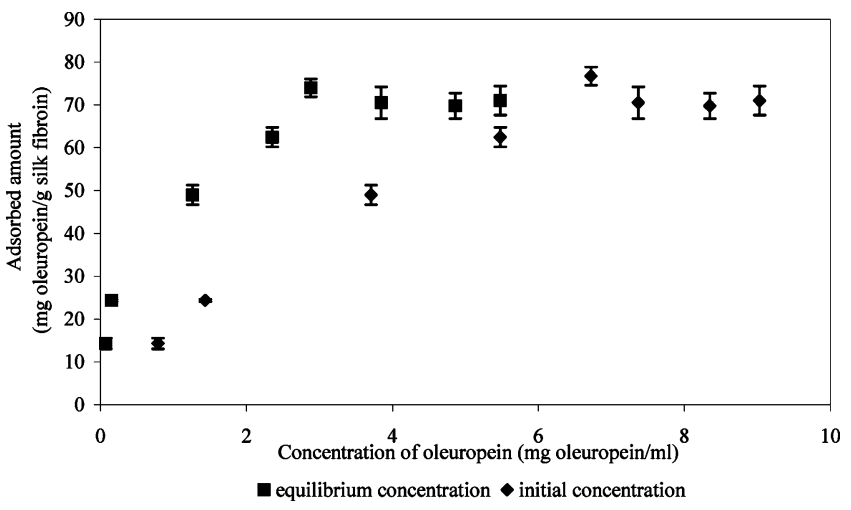

Figure 4. Adsorption behavior of oleuropein on silk fibroin at different initial concentrations.

Langmuir parameters of the adsorption isotherm were calculated by using the Langmuir equation

$$
q=\frac{q_{0} c}{K+c}
$$

where $q_{0}=$ maximum adsorbed amount ( $\mathrm{mg}$ of oleuropein $/ \mathrm{g}$ of silk) and $K=$ Langmuir constant (mg of oleuropein $/ \mathrm{mL}$ of water); $1 / q$ versus $1 / c$ was drawn, and the Langmuir parameters were calculated by using the linearization of the Langmuir equation. The intercept of this curve gives $1 / q_{0}$, whereas the slope of the curve is equal to $K / q_{0} .\left(1 / q_{0}=0.0138 ; q_{0}=72.46\right.$ $\mathrm{mg}$ of oleuropein/g of silk; $K / q_{0}=0.0041 ; K=0.297 \mathrm{mg} /$ $\mathrm{mL}$.) The maximum adsorption capacity of silk fibroin for oleuropein was calculated as $72.46 \mathrm{mg}$ of oleuropein/g of silk by using the Langmuir equation and assuming the monolayer coverage was valid. From the plateau of the adsorption isotherm given in Figure 4, the maximum adsorption capacity was determined as $71.02 \mathrm{mg}$ of oleuropein/g of silk. The proximity of these values with the model and experimental data proved the validity of the monolayer coverage.

Although rutin is the second most abundant compound in olive leaf extract, the rutin content of olive leaves is very low compared with the oleuropein content. For this reason, the adsorbed rutin amount on silk fibroin was lower compared to the adsorbed amount of oleuropein. First, the effect of initial concentration on the adsorption of rutin was observed, and its adsorption isotherm at $25^{\circ} \mathrm{C}$ was evaluated as shown in Figure 5.

The initial concentration of rutin was changed from 0.13 to $1.34 \mathrm{mg} / \mathrm{mL}$. In this range, its adsorption on silk fibroin was increased until its initial concentration was set to $0.88 \mathrm{mg}$ rutin/ $\mathrm{mL}$, and the adsorbed amount was nearly the same over this concentration.

The adsorption isotherm of rutin was evaluated within this range of initial concentration by using the equilibrium values of rutin in the solution and on the adsorbent. Similar results were observed as in the case of the oleuropein adsorption on silk fibroin. The experimental data better fit the Langmuir model than the Freundlich model. However, because of the low concentration values, $1 / q$ and $1 / c$ values of the linear part of the adsorption isotherm were used in the modeling instead of using all of the experimental data. The maximum adsorption capacity of silk fibroin for rutin was calculated as $16.47 \mathrm{mg}$ of rutin/g of silk from this curve, whereas the maximum adsorption capacity was determined as $13.5 \mathrm{mg}$ of rutin/g of silk from the plateau of the adsorption isotherm. These values demonstrate that the monolayer coverage did not achieve $100 \%$. Because it 


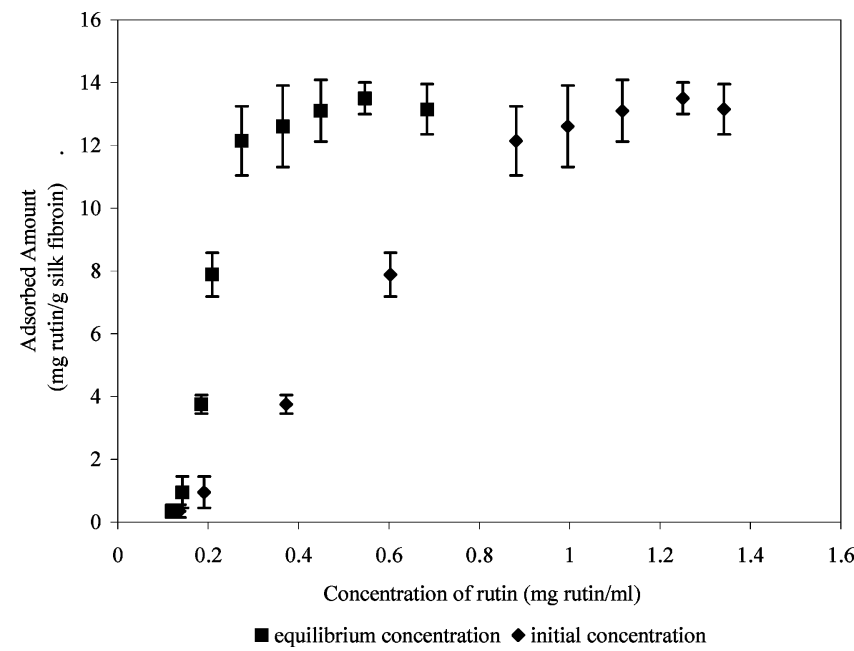

Figure 5. Adsorption behavior of rutin on silk fibroin at different initial concentrations.

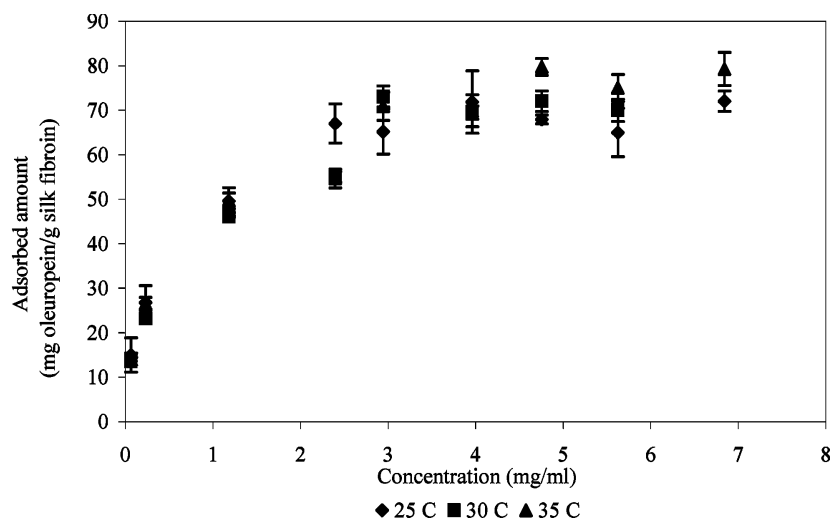

Figure 6. Effect of temperature on adsorption of oleuropein on silk fibroin.

was a multicomponent system, the surface of the silk fibroin was covered with not only rutin but also oleuropein.

Effect of Temperature on the Adsorption of Oleuropein and Rutin. Temperature is another parameter that affects the adsorption capacity of adsorbents. Adsorption was established at three different temperatures, 25,30 , and $35^{\circ} \mathrm{C}$. All of these experiments were run in water, and the solid/liquid ratio was always 1:20 to see only the effect of temperature on adsorption. The adsorption isotherms obtained at these three temperatures are demonstrated in Figure 6. A clear difference could not be detected between the adsorption capacities of silk fibroin at these three temperatures within the range of error margins. This was probably due to the proximity of temperatures, but it was not practical to perform the adsorption at lower than $25^{\circ} \mathrm{C}$ or higher than $35^{\circ} \mathrm{C}$ because of the economics concerns. There is also a risk of polyphenol degradation at temperatures above $40{ }^{\circ} \mathrm{C}$. The maximum adsorption capacity values of this process did not increase or decrease in any order. For this reason, $q_{0}$ values could not give any information about the nature of the process. Under these conditions, the equilibrium adsorption capacity of oleuropein was found to be $72.46 \mathrm{mg}$ of oleuropein/g of silk at $25{ }^{\circ} \mathrm{C}$.

The adsorption behavior of silk fibroin for rutin was also investigated at the temperatures of 25,30 , and $35^{\circ} \mathrm{C}$, which are demonstrated in Figure 7. A clear difference could not be detected between the adsorption capacities of silk fibroin at these three temperatures within the range of error margins. For this

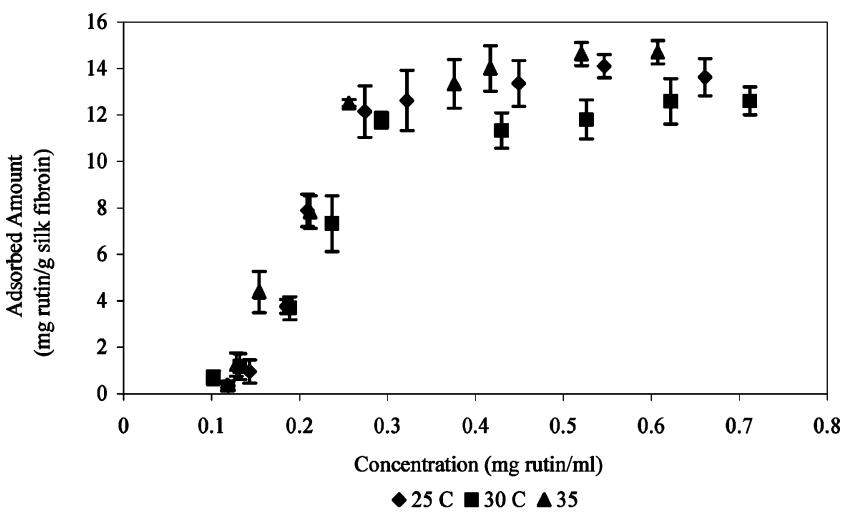

Figure 7. Effect of temperature on adsorption of rutin on silk fibroin.

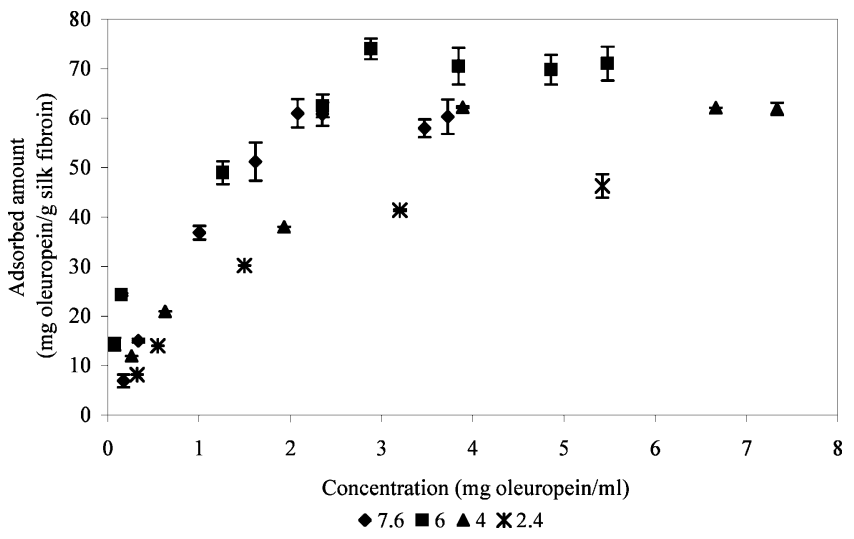

Figure 8. Effect of pH on adsorption of oleuropein on silk fibroin.

reason, the nature of the process cannot be understood, but 25 ${ }^{\circ} \mathrm{C}$ seems to be a favorable condition for the adsorption of rutin as well.

Effect of pH on Adsorption of Oleuropein and Rutin. The adsorption behavior of polyphenols may differ due to the $\mathrm{pH}$ of the medium. Therefore, experiments were run at $\mathrm{pH} 2.4,4.0$, 6.0, and 7.6. The $\mathrm{pH}$ of the system was adjusted to $2.4,4.0$, and 7.6 by using citric acid and phosphate buffer. The other experiment was run in deionized water, which was at a $\mathrm{pH}$ of 6.0. Figure 8 demonstrates the adsorption behavior of silk fibroin at these four different $\mathrm{pH}$ values.

The adsorptive properties of silk fibroin were closer to each other at all of the $\mathrm{pH}$ values, except at $\mathrm{pH} 2.4$. The adsorbed amount of oleuropein was significantly less at $\mathrm{pH} 2.4$, showing that the electrostatic interactions between the adsorbent and adsorbate were not as strong as the hydrophobic interactions. From the plateau of the adsorption isotherms, the maximum adsorbed amounts were found as $61,71,62$, and 45 for the $\mathrm{pH}$ values of 7.6, 6.0, 4.0, and 2.4, respectively. The adsorbed amount of oleuropein at $\mathrm{pH} 4.0$ was as nearly the same as that at $\mathrm{pH}$ 7.6. The maximum adsorption of oleuropein was achieved at $\mathrm{pH}$ 6.0. Similar results were reported for the adsorption of polyphenols. In the literature, the adsorption affinity of gelatinpolyvinyl alcohol for flavonol glycosides was found to be high between pH 5.0 and 6.5 (30). In the phenol adsorption, typically the amount adsorbed decreased at both low and high $\mathrm{pH}$ values, and the maximum adsorption values for the irreversible systems were generally obtained in unbuffered systems. The data fit well the Langmuir equation with high regression coefficients. The experimental data and the model values all represented that $\mathrm{pH}$ 6.0 was the best $\mathrm{pH}$ value for the adsorption of oleuropein.

Figure 9 demonstrates the adsorption isotherms of rutin at $\mathrm{pH} 7.6,6.0,4.0$, and 2.4. The adsorbed amounts of rutin were 


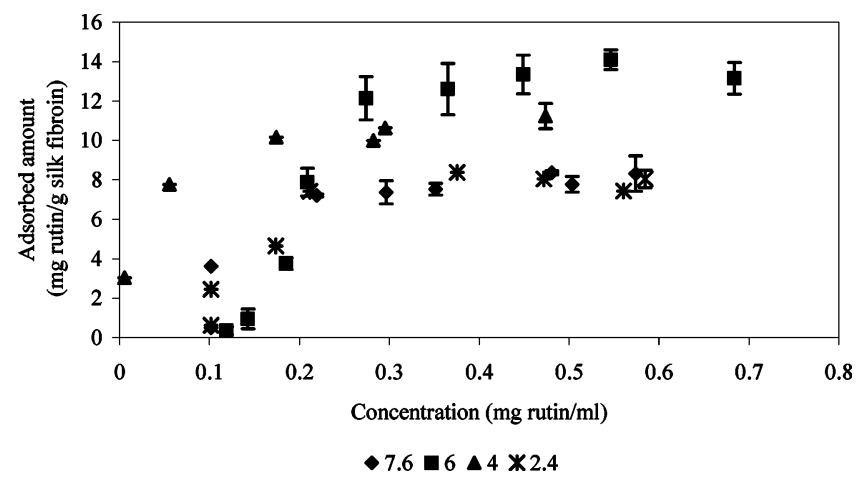

Figure 9. Effect of $\mathrm{pH}$ on adsorption of rutin on silk fibroin.

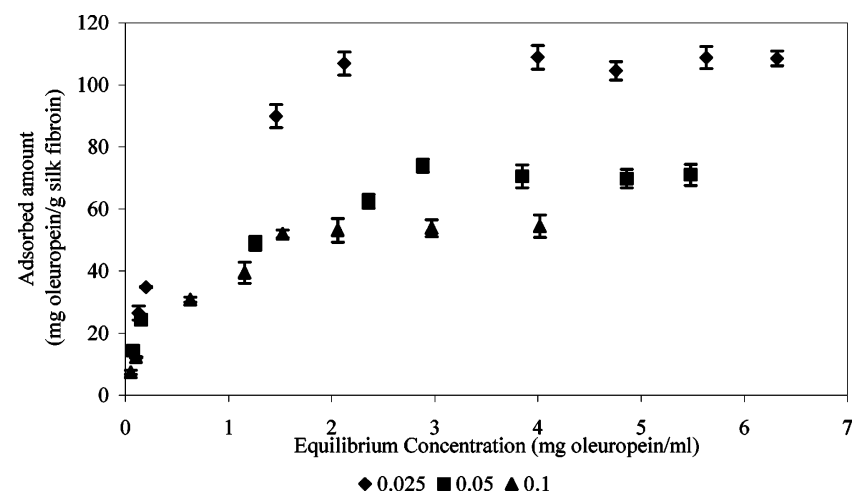

Figure 10. Effect of solid/liquid ratio on adsorption of oleuropein.

significantly less at $\mathrm{pH} 2.4$ and 7.6, showing that the electrostatic interactions were not the main interactions between the silk fibroin and rutin. From the plateau of the adsorption isotherms, the maximum adsorbed amounts were found as 8.3, 13.5, 10.8, and $7.5 \mathrm{mg}$ of rutin/g of silk fibroin for the $\mathrm{pH}$ values of 7.6, $6.0,4.0$, and 2.4 , respectively. The maximum adsorption was again accomplished at $\mathrm{pH} 6$ as in the case of oleuropein adsorption. The linear portion of the adsorption isotherms fit well the Langmuir equation with high regression coefficients. The maximum adsorption values calculated from the equation are higher than the maximum adsorbed values found from the plateau of the adsorption isotherms, showing that the monolayer coverage was not accomplished.

Effect of Solid/Liquid Ratio on Adsorption of Oleuropein and Rutin. The solid/liquid ratio is another important criterion in the adsorption from solution, which affects the economy of the system.

The adsorption behavior of oleuropein in water was observed in three different solid/liquid ratios at $25^{\circ} \mathrm{C}$ and $\mathrm{pH} 6.0$ (Figure 10). The solid/liquid ratio was adjusted to $0.025,0.05$, and 0.1 by changing only the adsorbent amount. The adsorption efficiency of oleuropein was increased with the decreasing adsorbent amount at constant initial concentrations of oleuropein. This might be due to the total removal of the solute even with low adsorbent concentration. Because the adsorbent was saturated when the solid/liquid ratio was 0.025 , the adsorbed amount per gram of adsorbent was lower when the solid/liquid ratio increased from 0.025 to 0.1 .

From the model, the maximum adsorption was found to be $117.65 \mathrm{mg}$ of oleuropein/g of silk fibroin, whereas the plateau of the adsorption isotherm gave this value as $108 \mathrm{mg}$ of oleuropein/g of silk fibroin. The adsorbed amount at $0.025 \mathrm{solid} /$ liquid ratio was 1.5 times the adsorbed amount at 0.05 solid/ liquid ratio, and it was nearly 2 times the adsorbed amount at $0.1 \mathrm{solid} /$ liquid ratio. Because of this, the decrease with the mass

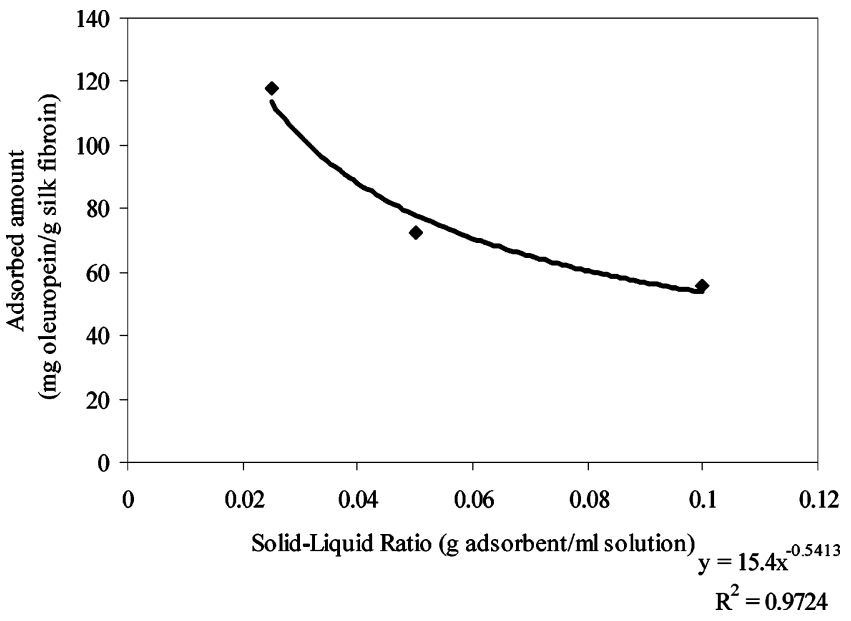

Figure 11. Adsorption efficiency of oleuropein at different solid/liquid ratios.

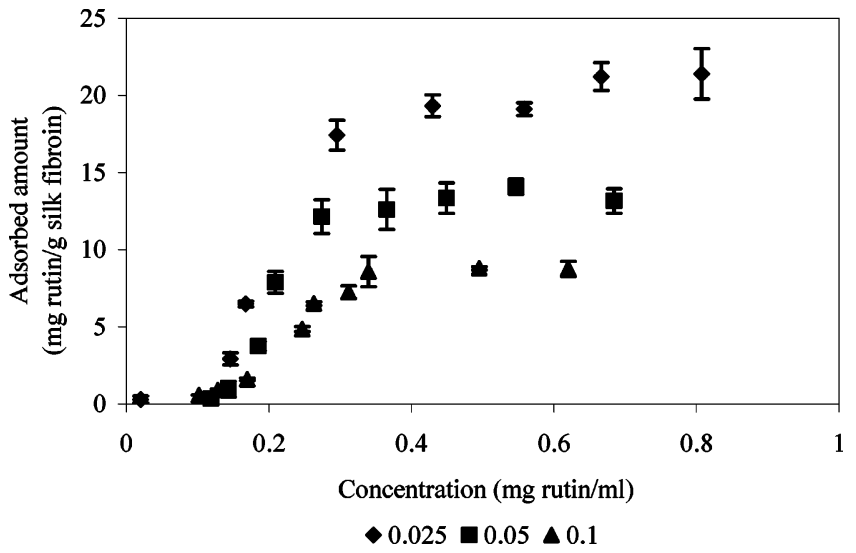

Figure 12. Effect of solid/liquid ratio on adsorption of rutin.

of the adsorbent can be represented by a power equation as given in Figure 11.

The adsorption of limonin and naringin on various natural and synthetic adsorbents also increased by a power equation as the solid/liquid ratio was decreased (16). Consequently, the maximum adsorption capacity of silk fibroin for oleuropein at $25^{\circ} \mathrm{C}$ in water was found as $108 \mathrm{mg}$ of oleuropein/g of silk fibroin when the solid/liquid ratio was 0.025 . To our knowledge, there have been no studies about the adsorption of polyphenols on silk fibroin or adsorption of olive leaf antioxidants on any adsorbents because of this; it was difficult to compare this value with other studies.

The solid/liquid ratio had inversely affected the adsorption of oleuropein on silk fibroin. Therefore, the rutin adsorption was also expected to decrease as the solid/liquid ratio was increased. The adsorbed amount of rutin was found when the solid/liquid ratio was $0.025,0.05$, and 0.1 at different initial concentrations. As is obvious in Figure 12, maximum adsorption efficiency was obtained when the solid/liquid ratio was 0.025 . This was probably due to the total removal of rutin with a low amount of silk fibroin. The maximum adsorbed amounts were determined as $21,13.5$, and $8.5 \mathrm{mg}$ of rutin/g of silk fibroin for solid/liquid ratios of $0.025,0.05$, and 0.1 , respectively.

There are slight differences in the maximum adsorption capacity of model and experimental data, showing that monolayer was not fully covered in this system. The solid/liquid ratio significantly affected the adsorption of rutin. Figure $\mathbf{1 3}$ represents the decrease in the adsorption efficiency with the mass of the adsorbent by a power equation. As a result, the maximum adsorption capacity of silk fibroin for rutin at $25{ }^{\circ} \mathrm{C}$ in water 


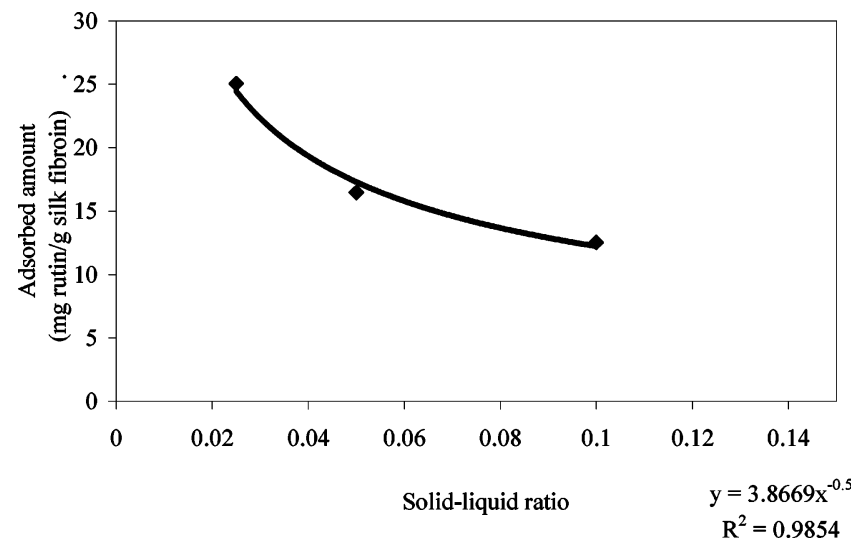

Figure 13. Adsorption efficiency of rutin at different solid/liquid ratios.

was found as $21 \mathrm{mg}$ of rutin/g of silk fibroin when the solid/ liquid ratio was 0.025 .

Because both rutin and oleuropein are adsorbed in this system, this system can be defined as a multicomponent adsorption system. The objective of adsorption in most real systems is to remove several adsorbates instead of one. This complicates the theoretical picture of equilibrium among adsorbates and adsorbent. The Langmuir model may be generalized from single to multicomponent adsorption. The Langmuir equation for multicomponent systems is given as

$$
\theta_{i}=\frac{(1 / K) C_{i}}{1+\sum_{i=1}^{n}(1 / K) C_{i}}
$$

where $\theta_{i}=$ surface coverage.

Using eq 2, one can estimate the fraction of the surface covered by oleuropein, rutin, or other polyphenols. However, this model is successful under the following conditions: (1) The competing components must obey the assumptions of the Langmuir-like equation for single solutes. (2) The capacity of the surface for each component must be the same. (3) Adsorbates must be competing for the same binding sites.

From eq 2, the surface coverages of both oleuropein and rutin were calculated as 0.29 and 0.66 . Because other polyphenols also adsorbed on silk fibroin, they covered the remaining 0.05 portion of available silk fibroin. The surface coverage of silk fibroin was also found by using the experimental data of rutin and oleuropein by assuming no other polyphenols were adsorbed on silk fibroin. In this case, the surface coverages of rutin and oleuropein were found as 0.16 and 0.84 . The difference between the surface coverage values of oleuropein and rutin was probably due to the differences in molecular sizes or differences in orientations of them on the surface of silk fibroin. Several researchers reported that if adsorption occurs on sites that were not equally accessible to all of the competing adsorbates, the Langmuir model was not expected to yield accurate results (31).

The adsorption affinity factors in most of the studies are defined as the equilibrium ratio in the initial linear region of the adsorption isotherms because the number of occupied sites is small as compared to the total active sites, which can be taken as constant and a measure of the affinity for the solute (16). The affinity factors for oleuropein and rutin were found from when the adsorption was performed at $25{ }^{\circ} \mathrm{C}$ and the solid/ liquid ratio was adjusted to 0.025 in water. The slopes of the initial linear region of these curves were determined as 60.06 for rutin and 50.41 for oleuropein, showing the fact that the adsorption affinity for rutin was higher than that for oleuropein. Although oleuropein content was higher than rutin, adsorption

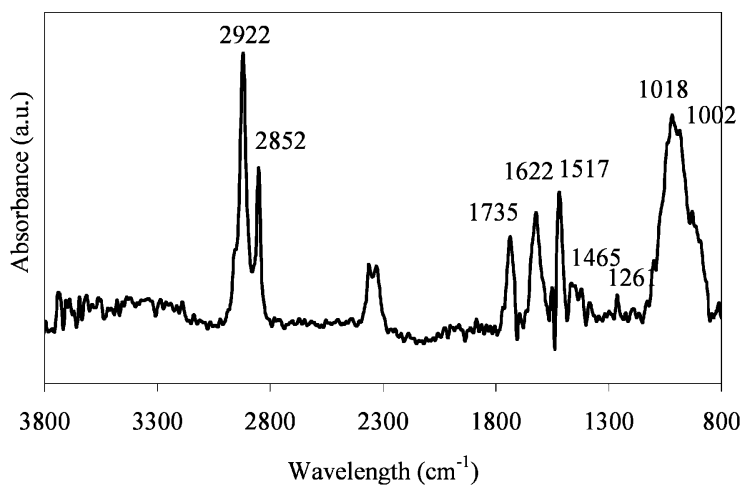

Figure 14. FTIR spectrum of adsorbed polyphenols on silk fibroin.

affinity for rutin was higher. This was probably due to the difference of the molecular weights between rutin and oleuropein. The molecular weight and molecular formula of rutin are 610.53 and $\mathrm{C}_{27} \mathrm{H}_{30} \mathrm{O}_{16}$, respectively. The molecular weight and molecular formula of oleuropein are 540.53 and $\mathrm{C}_{25} \mathrm{H}_{32} \mathrm{O}_{13}$, respectively (32). Increase in the molecular weight increased the interactions between biopolymers and polyphenols. Because the molecular weight of the rutin is higher than that of the oleuropein, the adsorption affinity for rutin is expected to be higher than that of oleuropein.

Consequently, the recoveries of oleuropein and rutin were increased from 54 and $65 \%$ to 80 and $88 \%$, respectively, by changing the $\mathrm{pH}$, solid/liquid ratio, and temperature. The solid/ liquid ratio was the most effective parameter in the adsorption of olive leaf antioxidants. As a result of this study, the purity of oleuropein and rutin was increased and polyphenol-rich silk fibroin was obtained.

Fourier Transform Infrared Spectroscopy (FTIR) of Adsorbed Polyphenols on Silk Fibroin. FTIR analyses were performed for silk fibroin. The main goal of this analysis was to investigate the change in the molecular conformation and orientation of silk fibroin before and after adsorption of polyphenols and examine whether the characteristic peaks of oleuropein can be seen on silk fibroin after adsorption. The IR spectrum of silk fibroin before adsorption was subtracted from the IR spectrum of silk fibroin after adsorption to see if any bands were left. In other words, the IR spectrum of silk fibroin was taken as background to understand which peaks belonged to the adsorbed polyphenols. In addition to this, analyses of olive leaf extract and oleuropein were performed to see which absorption bands were attributed to oleuropein in olive leaf extract. Figures 14, 15, and $\mathbf{1 6}$ give the FTIR spectra of adsorbed polyphenols on silk fibroin, olive leaf extract, and oleuropein, respectively.

The existence of absorption bands in the FTIR spectrum of adsorbed polyphenols proved the accumulation of oleuropein on the silk fibroin because absorption bands corresponding to oleuropein were present in the spectrum. The appearance of C-H stretching bands in the regions of 2852-2854 and 2922 $\mathrm{cm}^{-1}$ in the IR spectra of olive leaf extract and oleuropein showed that they were the characteristic bands of oleuropein. The observed peaks at 2922 and $2852 \mathrm{~cm}^{-1}$ in the spectrum of adsorbed polyphenols proved the adsorption of oleuropein on silk fibroin. The peaks at 1516 and $1517 \mathrm{~cm}^{-1}$ in the spectrum of olive leaf extract and adsorbed olive leaf polyphenols revealed the existence of aromatic rings. $\mathrm{C}-\mathrm{O}$ stretching in phenols produces an absorption band in the $1300-1100 \mathrm{~cm}^{-1}$ region. This band appeared at $1282 \mathrm{~cm}^{-1}$ in the IR spectrum of both the oleuropein and olive leaf extract, whereas it appeared at $1261 \mathrm{~cm}^{-1}$ in the adsorbed polyphenols. Furthermore, the $\mathrm{C}=$ 


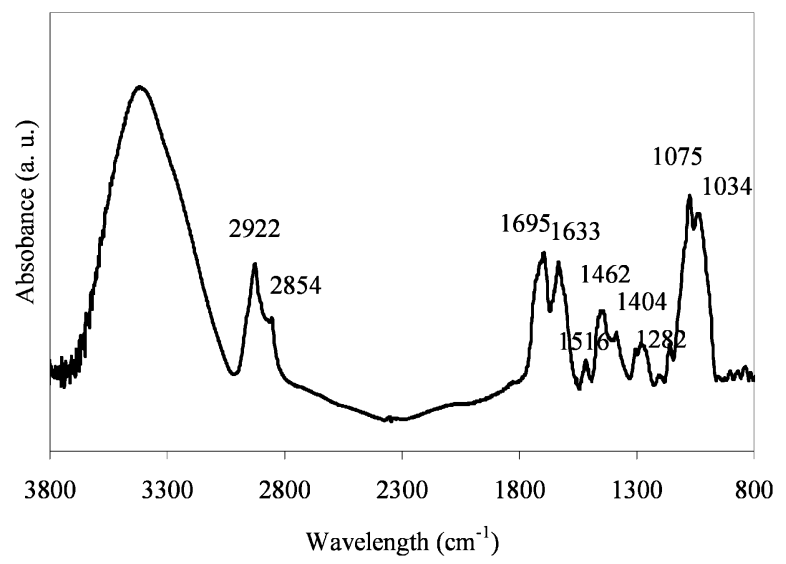

Figure 15. FTIR spectrum of olive leaf extract.

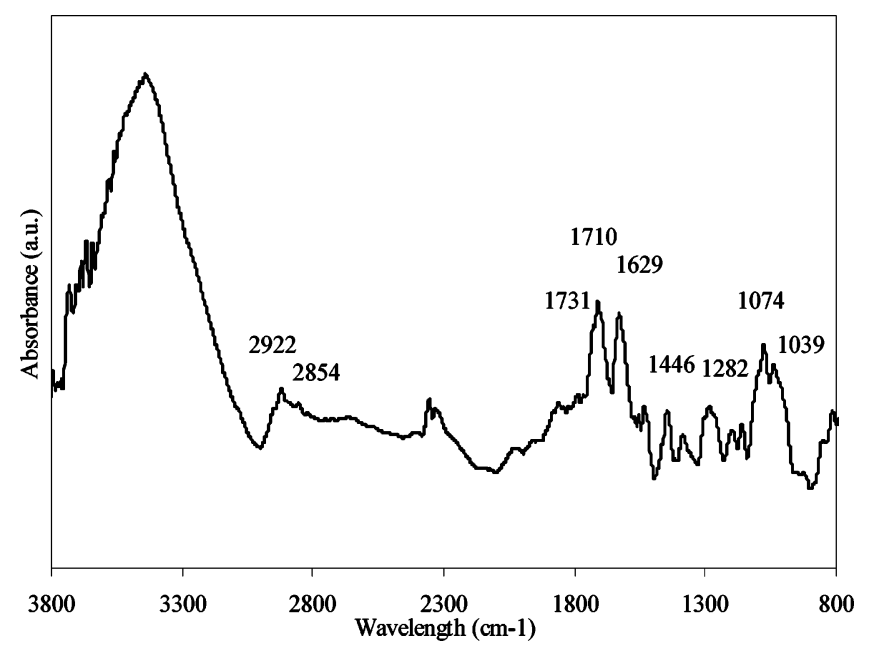

Figure 16. FTIR spectrum of oleuropein.

O stretching was observed at 1710 and $1731 \mathrm{~cm}^{-1}$ in the IR spectrum of oleuropein and at $1735 \mathrm{~cm}^{-1}$ in the spectrum of adsorbed polyphenols. As a result, the observed principal bands in the spectra of oleuropein and olive leaf extract were also observed in the spectrum of adsorbed polyphenols, proving the oleuropein and rutin adsorption on silk fibroin.

Antioxidant Analysis of Adsorbed Polyphenols on Silk Fibroin. The aim of this study was to produce a protein-based functional food having antioxidative and antimicrobial properties. In accordance with this aim, it should be known whether olive leaf polyphenols show their functionality after adsorption or not. Because it was impossible to find the antioxidant capacity of the solid phase including silk fibroin and olive polyphenols, it was dissolved in Ajisawa's reagent to determine the antioxidant activity. Four samples were analyzed in a UV-visible spectrophotometer. The first sample was the Ajisawa solution itself, the second sample was the olive leaf extract solution containing the same amount of adsorbed polyphenols, the third sample was the adsorbed polyphenols and silk fibroin, and the fourth sample was the silk fibroin solution alone. Ajisawa's reagent did not exhibit any antioxidative activity, and it did not induce the antioxidant activity of the samples. The antioxidant activities of these samples were determined in terms of TEAC (millimoles per gram).

Silk fibroin alone also exhibited antioxidant activity toward the ABTS radical cation, and its antioxidant capacity was increased from 1.93 to $3.61 \mathrm{TEAC} \mathrm{mmol} / \mathrm{g}$ after adsorption. On the other hand, the olive leaf extract containing the same amount of adsorbed polyphenols exhibited $0.88 \mathrm{TEAC} \mathrm{mmol} /$
Table 1. Results of Antimicrobial Tests

\begin{tabular}{lcccc}
\hline & \multicolumn{4}{c}{ inhibition zone for bacteria (mm) } \\
\cline { 2 - 5 } & $\begin{array}{l}\text { E. } \\
\text { coli }\end{array}$ & $\begin{array}{c}\text { K. pneu- } \\
\text { moniae }\end{array}$ & $\begin{array}{c}\text { S. } \\
\text { aureus }\end{array}$ & $\begin{array}{c}\text { P. aerug- } \\
\text { inosa }\end{array}$ \\
\hline $\begin{array}{l}\text { olive leaf extract (OLE) } \\
\text { physical mixture of }\end{array}$ & & 19 & \\
$\quad$ OLE and SF & & 20 & \\
silk fibroin (SF) & & & & \\
adsorbed OLE on SF & & 16 & 20 & \\
gentamicin & 24 & 8 & 20 & 21 \\
penicillin & & & 44 & \\
vancomycin & & & 14 & \\
\hline
\end{tabular}

g, a lower antioxidant activity compared to the adsorbed polyphenols on silk fibroin. The amount of oleuropein and rutin in adsorbed polyphenols on silk fibroin was higher compared to the amount in olive leaf extract. Therefore, they exhibited higher antioxidant activity.

In this study, antioxidative activity was assessed with TEAC in solution. Although this is a valid in vitro/ex situ method, in situ methods such as electron spin resonance (ESR) studies using 1,1-diphenyl-2-picrylhydrazyl (DPPH) in biomembrane are well suited for the olive leaf extract treated silk fibroin. Results from the ESR could yield more near-to-reality data, given the fact that treated biomaterials would probably be used in the form of solids rather than liquid (or solution). In the literature, free radical scavenging reactions of green tea polyphenols were successfully investigated with ESR spectroscopy in the phospholipids bilayer of liposomes using the DPPH radical as model (33).

Antimicrobial Tests of Adsorbed Polyphenols on Silk Fibroin. The antimicrobial activities of silk fibroin (SF), olive leaf extract (OLE), a physical mixture of olive leaf extract with silk fibroin, and silk fibroin containing adsorbed polyphenols were investigated toward four different bacteria, Escherichia coli, Staphylococcus aureus, Pseudomonas aeruginosa, and Klebsiella pneumoniae. Furthermore, the effects of three different commercial antibiotics, gentamicin, penicillin, and vancomycin, toward these bacteria were studied as positive control. The samples and antibiotics were all in pellet form with 14 and $6 \mathrm{~mm}$ diameters, respectively. After 1 day, inhibition zones around the samples and antibiotics were observed. However, a color change was also observed around the olive leaf extract containing samples, which showed the diffusion of the polyphenolic structures. The inhibition zones of samples and antibiotics are given in Table 1. All of the samples except silk fibroin demonstrated antimicrobial activities. Samples were generally resistant toward $S$. aureus. Only the adsorbed polyphenols on silk fibroin inhibited the growth of $K$. pneumoniae among the samples.

The measured inhibition zone of adsorbed OLE on SF was the same with the physical mixture of OLE and SF. Thus, adsorption did not have any inverse effect on the antimicrobial activity of polyphenols. The inhibition effect of adsorbed OLE on SF toward $K$. pneumoniae probably was due to the selective adsorption of oleuropein. It was known that oleuropein was the significant compound in the antimicrobial activity of olive leaves. Because the amount of oleuropein on SF was high after adsorption, this sample hindered the growth of $K$. pneumoniae. $S$. aureus is a Gram-positive bacterium, whereas other bacteria used in this study are Gram-negative. The Gram-positive bacteria are more sensitive to antimicrobial agents because they do not have an outer membrane for their cell membranes. Because the effect of olive leaf polyphenols against Gram-positive bacteria 
is higher compared to that of the Gram-negative bacteria, they are probably causing inhibition of cell membrane function.

As a result, silk fibroin with oleuropein and rutin can be used as a functional food because of their antimicrobial activity. Nowadays, there is a growing concern about the quality and safety of foods. Therefore, the use of these bioactive phytochemicals combined with protein as natural preservatives to control pathogenic growth and to eliminate microbial contamination can probably be preferred to the use of synthetic preservatives in food preservation.

Desorption of Olive Leaf Antioxidants. The isolation and purification of antioxidants are desired processes for the food, cosmetics, and pharmacological industries. New and low-cost commercial techniques have been researched especially for their use in dietary supplements. In this part of the study, the adsorption of oleuropein and rutin was accomplished in high percentages. These compounds continued to exhibit their antimicrobial and antioxidative properties on the silk fibroin. If desorption of olive leaf antioxidants could be successfully achieved, then they would be recovered in large amounts from crude olive leaf extract. To desorb these compounds, desorption was performed in $70 \%$ aqueous ethanol solution. The desorption behavior of oleuropein and rutin was followed during 4 days. The system reached equilibrium within $20 \mathrm{~h}$. The maximum adsorption amounts of rutin and oleuropein (when the solid/ liquid ratio was 0.025 ) were $21 \mathrm{mg}$ of rutin/g of silk fibroin and $108 \mathrm{mg}$ of oleuropein/g of silk fibroin. In the desorption process, $17 \mathrm{mg}$ of rutin/g of silk fibroin and $91.8 \mathrm{mg}$ of oleuropein/g of silk fibroin were recovered from the adsorbent. Thus, $81 \%$ of rutin and $85 \%$ of oleuropein were successfully removed from the adsorbent surface. Therefore, $1 \mathrm{~g}$ of silk fibroin can provide the removal of $69 \%$ of oleuropein and $93 \%$ of rutin from $1 \mathrm{~g}$ of olive leaf extract. After desorption, the color of the silk fibroin again turned to light yellow.

The antioxidant capacity of desorbed polyphenols was analyzed to check whether they lost their antioxidant properties after the desorption process or not. The results were compared with the antioxidant capacities of the olive leaf extract containing the same amount of desorbed polyphenols. The antioxidant activity of desorbed polyphenols was nearly 2 times higher than that of the olive leaf extract (containing the same amount of desorbed polyphenols). The antioxidants have a synergistic effect on each other when they are found together, which is why the purification of highly bioactive oleuropein and rutin caused the observation of an increased antioxidant capacity. The antioxidant capacity of desorbed olive leaf antioxidants was found to be $1.5 \mathrm{TEAC} \mathrm{mmol} / \mathrm{g}$.

Conclusions. In this study, we explored the potential use of silk fibroin for the recovery of polyphenols as an adsorbent. The experimental data of adsorption isotherms were well fitted to a Langmuir model. The maximum adsorption capacities at these conditions were calculated as $108 \mathrm{mg}$ of oleuropein/g of silk fibroin and $21 \mathrm{mg}$ of rutin/g of silk fibroin. The adsorption of olive leaf antioxidants on silk fibroin was confirmed by FTIR. Silk fibroin alone showed antioxidative activity, whereas it did not exhibit any antimicrobial activity. After adsorption of olive leaf antioxidants, the antioxidative property of silk fibroin increased. It gained antimicrobial activity against $S$. aureus and K. pneumoniae.

Silk fibroin was determined as a promising protein-based biopolymer in the production of antioxidative and antimicrobial functional food or dietary supplement after adsorption of olive leaf antioxidants. Furthermore, it can also be used in the purification of olive leaf antioxidants from the crude olive leaf extracts.

\section{LITERATURE CITED}

(1) Aehle, E.; Grandic, S. R.; Ralainirina, R.; Baltora-Rosset, S.; Mesnard, F.; Prouillet, C.; Maziere, J.; Fliniaux, M. Development and evaluation of an enriched natural antioxidant preparation obtained from aqueous spinach (Spinacia oleracea) extracts by an adsorption procedure. Food Chem. 2004, 86, 579-585.

(2) Bonilla, M.; Salido, S.; Beekb, T.; Linares-Palomino, P. J.; Altarejos, J.; Nogueras, M.; Sanchez, A. Isolation and identification of radical scavengers in olive tree (Olea europaea) wood. J. Chromatogr. 2006, 1112, 311-318.

(3) Sun, J.; Chu, Y. F.; Wu, X.; Liu, R. H. Antioxidant and antiproliferative activities of common fruits. J. Agric. Food Chem. 2002, 50, 7449-7454.

(4) Chu, Y. F.; Sun, J.; Wu, X.; Liu, R. H. Antioxidant and antiproliferative activities of common vegetables. J. Agric. Food Chem. 2002, 50, 6910-6916.

(5) Cowan, M. M. Plant products as antimicrobial agents. Clin. Microbiol. Rev. 1999, 12, 564-582.

(6) Al-Azzawie, H. F.; Saeed Alhamdani, M. S. Hypoglycemic and antioxidant effect of oleuropein in alloxan-diabetic rabbits. Life Sci. 2006, 78, 1371-1377.

(7) Garcia, O. B.; Castillo, J.; Lorente, J.; Ortuno, A.; Del Rio, J. A. Antioxidant activity of phenolics extracted from Olea europaea L. leaves. Food Chem. 2000, 68, 457-462.

(8) Ranalli, A.; Contento, S.; Lucera, L.; Febo, M.; Archegiani, D.; Fonzo, V. Factors affecting the contents of iridoid oleuropein in olive leaves (Olea europaea L.). J. Agric. Food Chem. 2006, $54,434-440$.

(9) Ghica, M. E.; Brett, A. M. O. Electrochemical oxidation of rutin. Electroanalysis 2005, 17, 313-318.

(10) Kurisawa, M.; Chung, J. E.; Uyama, H.; Kobayashi, S. Enzymatic synthesis and antioxidant properties of poly(rutin). Biomacromolecules 2003, 4, 1394-1399.

(11) Sheu, J. R.; Hsiao, G.; Chou, P. H.; Shen, M. Y.; Chou, D. S. Mechanisms involved in the antiplatelet activity of rutin, glycoside of the flavonol quercetin, in human platelets. J. Agric. Food Chem. 2004, 52, 4414-4418.

(12) Shi, J.; Yu, J.; Pohorly, J.; Young, J. C.; Bryan, M.; Wu, Y. Optimization of the extraction of polyphenols from grape seed meal by aqueous ethanol solution. Food, Agric. Environ. 2003, $1,42-47$.

(13) Koleva, I. I.; Beek, T. A.; Linssen, J. P. H.; Groot, A.; Evstatieva, L. N. Screening of plant extracts for antioxidant activity: a comparative study on three testing methods. Phytochem. Anal. 2002, 13, 8-17.

(14) Yoon, S. Y.; Choi, W. J.; Park, J. M.; Yang, J. W. Selective adsorption of flavonoid compounds from the leaf extract of Ginkgo biloba L. Biotechnol. Tech. 1997, 11, 553-556.

(15) Scordino, M.; Mauro, A.; Passerini, A.; Maccarone, E. Adsorption of flavonoids on resins: cyanidin 3-glucoside. J. Agric. Food Chem. 2004, 52, 1965-1972.

(16) Ribeiro, M. H.; Silveira, D.; Ferreira-Dias, S. Selective adsorption of limonin and naringin from orange juice to natural and synthetic absorbents. Eur. Food Res. Technol. 2002, 215, 462-471.

(17) Tang, H. R.; Covington, A. D.; Hancock, R. A. Structure-activity relationships in the hydrophobic interactions of polyphenols with cellulose and collagen. Biopolymers 2003, 70, 403-413.

(18) Takagai, Y.; Kubota, T.; Kobayashi, H.; Tashiro, T.; Takahashi, A.; Igarashi, S. Adsorption and desorption properties of transresveratrol on cellulose cotton. Anal. Sci. 2005, 2, 183-186.

(19) Lee, K. G.; Kweon, H. Y.; Yeo, J. H.; Woo, S. O.; Lee, J. H.; Park, Y. H. Structural and physical properties of silk fibroin/ alginate blend sponges. J. Appl. Polym. Sci. 2004, 93, 21742179. 
(20) Altman, G. H.; Diaz, F.; Jakuba, C.; Calabro, T.; Horan, R. L.; Chen, J.; Lu, H.; Richmond, J.; Kaplan, D. L. Silk-based biomaterials. Biomaterials 2003, 24, 401-416.

(21) Park, S. J.; Lee, K. Y.; Ha, W. S.; Park, S. Y. Structural changes and their effect on mechanical properties of silk fibroin/chitosan blends. J. Appl. Polym. Sci. 1999, 74, 2571-2575.

(22) Bayraktar, O.; Malay, Ö.; Özgarip, Y.; Batgün, A. Silk fibroin as a novel coating material for controlled release of theophylline. Eur. J. Pharm. Biopharm. 2005, 60, 373-381.

(23) Coulombre, J.; Moore, W. The adsorption of sulfanilamide on silk fibroin. J. Am. Chem. Soc. 1949, 71, 661-664.

(24) Pauling, L. The adsorption of water by proteins. J. Am. Chem. Soc. 1945, 67, 555-557.

(25) Kongkachuichaya, P.; Shitangkoonb, A.; Chinwongamorn, N. Thermodynamics of adsorption of laccaic acid on silk. Dyes Pigm. 2002, 53, 179-185.

(26) Re, R.; Pellegrini, N.; Proteggente, A.; Pannala, A.; Yang, M.; Rice-Evans, C. Antioxidant activity applying an improved ABTS radical cation decolorization assay. Free Radical Biol. Med. 1999, $26,1231-1237$

(27) Yamada, H.; Nakao, H.; Takasu, Y.; Tsubouchi, K. Preparation of undegraded native molecular fibroin solution from silkworm cocoons. Thin Solid Films 2001, 440, 208-216.

(28) Kim, M. R.; Kim, W. C.; Lee, D. Y.; Kim, C. W. Recovery of narirutin by adsorption on a non-ionic polar resin from a waterextract of Citrus unshiu peels. J. Food Eng. 2007, 78, 2732.
(29) Liao, X. P.; Lu, Z. B.; Shi, B. Selective adsorption of vegetable tannins onto collagen fibers. Ind. Eng. Chem. Res. 2003, 42, 3397-3402.

(30) Xu, M.; Shi, Z.; Shi, R.; Liu, J.; Lu, Y.; He, B. Synthesis of the adsorbent based on macroporous copolymer MA-DVB beads and its application in purification for the extracts from Ginkgo biloba leaves. React. Funct. Polym. 2000, 43, 297-304.

(31) Alkhamis, K. A.; Wurster, D. E. Prediction of adsorption from multicomponent solutions by activated carbon using single-solute parameters, part II-proposed equation. Am. Assoc. Pharm. Sci. 2002, 23, 1-8.

(32) Walter, W. M.; Fleming, H. P.; Etchells, J. L. Preparation of antimicrobial compounds by hydrolysis of oleuropein from green olives. Appl. Microbiol. 1973, 26, 773-776.

(33) Chen, C.; Tang, H. R.; Sutcliffe, L. H.; Belton, P. S. Green tea polyphenols react with 1,1-diphenyl-2-picrylhydrazyl free radicals in the bilayer of liposomes: direct evidence from electron spin resonance studies. J. Agric. Food Chem. 2000, 48, 57105714.

Received for review October 2, 2006. Revised manuscript received December 3, 2006. Accepted December 7, 2006. We thank the Turkish State Planning Organization and Natural Products Research Development Unit (NPRDU) located in the Izmir Technology Development Zone for their financial supports.

JF0628290 